\title{
PRIME - A Passive Radar Sounding Concept for Io
}

G. Steinbrügge ${ }^{1}$, A. Romero-Wolf ${ }^{2}$, S.T. Peters ${ }^{1}$, D.M. Schroeder ${ }^{1}$, L. Carrer ${ }^{3}$, C.W. Hamilton ${ }^{4}$, L. Carter $^{4}$, C. J. Bierson ${ }^{5}$, D.D. Blankenship ${ }^{6}$, C. Chan ${ }^{6}$, L. Fanara ${ }^{7}$, C. Grima ${ }^{6}$, H. Hay ${ }^{2}$, H. Hussmann $^{7}$, J.T. Keane ${ }^{2}$, M. Maurice ${ }^{7}$, A. Nikolaou ${ }^{8}$, Y. Rosas-Ortiz ${ }^{7}$, K.M. Scanlan ${ }^{6}$, K.M. Soderlund $^{6}$, A. Stark ${ }^{7}$, I. Varatharajan 7 , J.R.C. Voigt ${ }^{4}$, and D.A. Young ${ }^{6}$

${ }^{1}$ Department of Geophysics, Stanford University, Stanford, CA, USA

${ }^{2}$ Jet Propulsion Laboratory, California Institute of Technology, CA, USA

${ }^{3}$ Department of Information Engineering and Computer Science, University of Trento, Italy

${ }^{4}$ Lunar and Planetary Laboratory, University of Arizona, Tucson, AZ, USA

${ }^{5}$ University of California Santa Cruz, Santa Cruz, CA, USA

${ }^{6}$ Institute for Geophysics, University of Texas at Austin, Austin, TX, USA

${ }^{7}$ Institute for Planetary Research, German Aerospace Center (DLR), Berlin, Germany

${ }^{8}$ Department of Physics, La Sapienza University of Rome, Rome, Italy

Corresponding author:

Gregor Steinbrügge

Email: gbs@stanford.edu

Phone: (650) 497.3498

Address: Department of Geophysics, 397 Panama Mall, Stanford University, Stanford, CA 943052215 USA 
In this white paper, we argue for a Passive Radar Io Magma Explorer (PRIME) to study Jupiter's moon Io as one of the priority targets of the in the next decade ${ }^{1,2}$. Jupiter is the second strongest source of radio noise within our Solar System, after the Sun itself. As passive radar sounding has the potential to operate with low power consumption and robust hardware, which is crucial in an environment like Jupiter, PRIME can also address major outstanding scientific questions regarding the crustal structure of Io.

\section{$\underline{1 . \text { Science Objectives }}$}

Io is subject to intense tidal heating leading to extensive volcanism, including intrusions and both effusive and explosive eruptions. The distribution of heating within Io, the potential existence of a global magma ocean, magma composition, and its ascent mechanisms remain a subject of debate $^{3}$. A window into the interior adds a third dimension on how we understand this fascinating satellite.

\subsection{Magmatic storage types}

The underlying magmatic storage region that sources volcanic eruptions on Io remains poorly constrained. While the volcanic activity is a direct consequence of excessive tidal heating, the location where the heat is generated within the interior is not yet understood. Shallow heating in the asthenosphere would have the tendency to concentrate dissipation at low latitudes whereas dissipation in the deeper interior would lead to more heat at the poles ${ }^{4,5}$. However, correlations between models favoring either mechanism with maps of Io's volcanic activity, do not favor any of the two end-member hypotheses ${ }^{6,7}$. It is therefore hypothesized that the ascent and distribution of magma in Io's interior is more complex than currently assumed by models. Magmas reaching the surface of a planetary body may be directly sourced from the mantle or from crustal storage reservoirs (i.e., magma chambers). On Io, erupted magma appears to have high temperatures and low viscosity, implying mafic to ultramafic compositions ${ }^{8}$. Eruption styles vary from voluminous outpourings of lava to explosive eruptions that generate lava fountains and large plumes. Io also includes hundreds of paterae, which may be similar to terrestrial calderas that form when large volumes of magma are erupted from shallow magma chambers, leading to collapse of the overburden. Prometheus patera may represent an example of this process, with magma supplied from a relatively shallow magma chamber, with a roof located between a depth of $\sim 2-3 \mathrm{~km}$ and $\sim 14 \mathrm{~km}^{9}$. Passive radar sounding would enable the detection of shallow magma reservoirs within Io's crust, helping to distinguish if magmas on Io are directly erupted from the mantle or pass through shallow magmatic reservoirs. This is fundamentally important because magma temperature is often considered to be a proxy for composition (mafic or ultramafic) and deeply sourced magma erupted adiabatically from greater depth would be hotter, but not necessarily of ultramafic compositions, whereas hotter magma at equilibrium with lower pressure conditions in the crust would imply more primitive compositions.

\subsection{Paterae}

Ionian paterae may be analogous to terrestrial calderas. Some patera on Io are greater than $200 \mathrm{~km}$ in diameter, with a mean diameter of $43 \mathrm{~km}^{10}$. However, it is unknown whether they form when a partially evacuated magma chamber collapses and would hence be compelling targets for radar sounding. 


\subsection{Thrust faulting}

Io's resurfacing rate is estimated to be on the order of $1 \mathrm{~cm} /$ year ${ }^{11}$. As each layer gets buried deeper, the area decreases leading to compressional stresses, which should lead to tectonic activity, and eventually forming Io's mountains by thrust faulting ${ }^{12,13}$. Radar sounding may be able to test this hypothesis by identifying subsurface thrust faults near mountains.

\subsection{Sulfur cycling}

Sulfur seems to play a significant role in Io's volcanic activity. However, models suggest that the sulfur in the interior should be depleted by now, considering the intense volcanism on Io over long time scales ${ }^{14}$. It is therefore likely that the sulfur will be recycled into the interior where it would be heated and may liquify. It has been proposed that the behavior of sulfur in Ionian magmas is analogous to terrestrial tholeiites where magmatic sulfur reaches saturation and exsolves as an immiscible sulfide. If the liquid sulfur layer could be detected as a function of latitude, it would represent an isothermal and therefore be extremely useful to constrain latitudinal tidal patterns and understand the thermal structure of Io's crust.

\subsection{Total electron content}

The ionosphere of Io is a measure for the frequency-dependent energy absorption of Io's atmosphere with important implications for atmospheric stability and composition. A passive receiver can cover frequencies of hundreds of $\mathrm{kHz}$ up to $35 \mathrm{MHz}$ without significantly driving the mass and volume requirements, which enables additional observation capabilities to study the total electron content of Io's ionosphere.

\section{Passive Sounding}

By eliminating the need to actively transmit a signal for echo detection, a passive radar that uses external sources for echo detection presents a low-resource radio sounding approach. For subJovian operation, the concept of passive radar sounding has been previously suggested in the context of Ganymede and Europa ${ }^{15-17}$ where a receiver from orbit, or on a ground-based lander, receive the direct and subsurface reflected signals from Jupiter in the radio frequency range. Passive sounding for scientific purposes has been successfully performed by Peters et al. ${ }^{18}$ with a prototype instrument on the side of a cliff using Sun's direct and reflected path off the ocean and extended in 2019 to subsurface sounding of Greenlandic ice sheets.

\section{Instrument Design}

Receivers for passive sounding are much simpler than a system needed for active sounding. Since the antenna does not need to transmit a signal, it can be significantly smaller than halfwavelength needed to achieve resonance. For a receive-only system, the antenna length can be electrically short since the system only needs to be efficient enough to be galactic noise limited compared to a typical internal system noise of $\sim 400 \mathrm{~K}$. JPL has designed, built, and successfully flown the DARPA High Frequency Research (DHFR) testbed using the principles of electrically short antenna receiver design ${ }^{18}$. JPL has also recently completed an Extended Phase A mission design for SunRISE ${ }^{19}$ based on a $6 \mathrm{U}$ cubesat (Figure 1) to localize coronal mass ejections for the Sun using a galactic noise-limited receiver on a smallsat with a $5 \mathrm{~m}$ total length dipole antenna. A 
passive sounder for Io would be similar to this and the antenna could be made even shorter since Jovian bursts are many thousands of times stronger than the Galactic background radiation.

JPL is also currently funding an internal research and technology development program to partially process passive sounding data on board a spacecraft. Continuously recording long traces of radio signals is data intensive and would likely dominate the telemetry allocation of a mission. By partially processing data on-board using a field-programmable gate array (FPGA), with traces of roughly one millisecond, the data rates are reduced by several orders of magnitude. Short times used for data reduction still enable ground processing to account for delay tracking (e.g., synthetic aperture formation) and radio frequency interference (RFI) rejection.

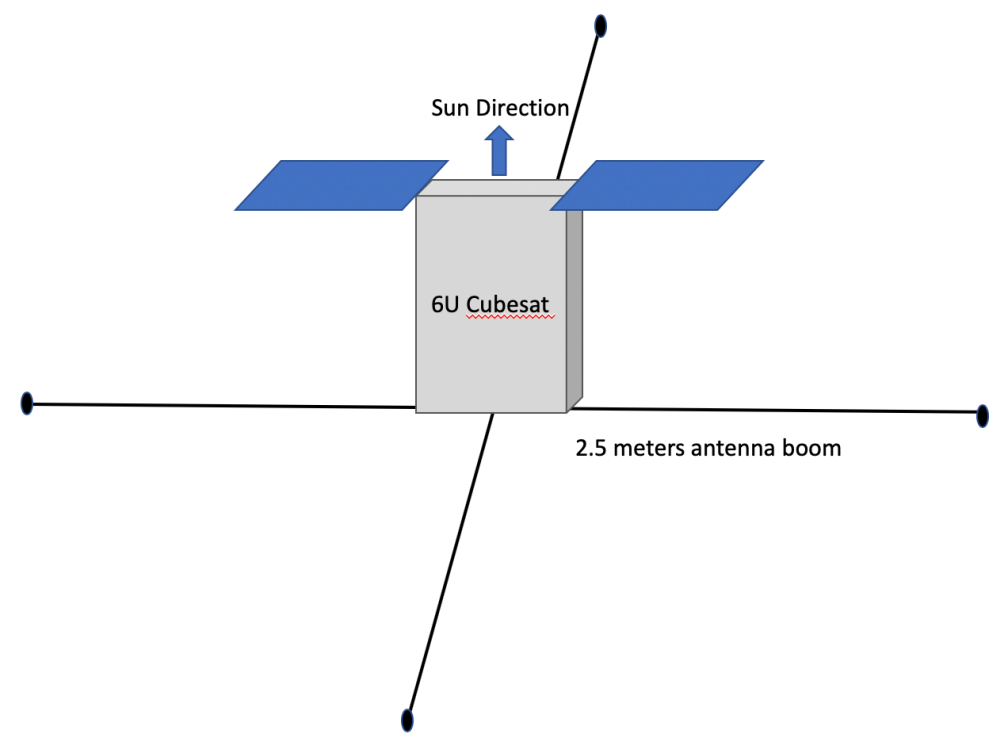

Figure 1: Passive radar concept accommodated by a $6 U$ CubeSat.

Io offers an ideal
situation to demonstrate
the passive sounding
technology for future
missions. Although the
Radar for Icy Moon
Exploration (RIME) and
the Radar for Europa
Assessment and Sounding:
Ocean to Near-Surface
(REASON) are including
passive sounding
capability, the
demonstrations are, to date,
not officially part of the
mission objectives.

Io offers an ideal situation to demonstrate the passive sounding technology for future missions. Although the Radar for Icy Moon Exploration (RIME) and the Radar for Europa Assessment and Sounding: Ocean to Near-Surface (REASON) are including passive sounding capability, demonstrations are, to date, mission objectives.

They also do not have real-time data processing capabilities built into them, which would be an essential component for future missions using a passive sounder. A passive sounder could be implemented as an instrument embedded on a spacecraft studying Io or as an independent flight element (e.g., as a CubeSat). Such a mission would be the ideal candidate in a challenging radiation environment like Jupiter.

A significant advantage of the more efficient design is the potential deployment and simultaneous use of multiple platforms (Figure 2). Using a multi-static passive system would enable 3D imaging of the subsurface which would be particularly valuable to resolve magma chambers and channels. While it is possible to perform this data processing with a single spacecraft, it would require an orbiter that operates for a longer period of time and records multiple parallel tracks with a crosstrack distance of only a few kilometers. Such an approach would be unrealistic for Io, since the lifespan of any craft in orbit is limited to a few days, given the severe radiation environment.

\section{$\underline{\text { 4. Jovian Noise Sources }}$}

Jovian radio noise (Figure 3) sources from charged particles that are deflected and accelerated by Jupiter's magnetic field. The particles move in and spiral around magnetic field lines towards 
either the south or the north pole leading to cyclotron emissions. For charged particles moving in Jupiter's magnetic field, the energy is such that radio waves are generated.

\subsection{Burst intensity}

To be useful for passive sounding, the flux density needs to be above the galactic noise background depicted in Figure 3. This is certainly true for the decametric (DAM) radio waves with frequencies between 10 and $40 \mathrm{MHz}$ and the hectometric radio waves (HOM) between 1 and $10 \mathrm{MHz}$; both exceed the galactic background and the solar noise by 5 orders of magnitude (Figure 3 ). For the purpose of reaching maximum penetration depth, lower frequencies are preferred. However, the lowest frequency for passive radar sounding of Io will be limited by the ionosphere of Io, where radio waves below the plasma cutoff frequency cannot penetrate to Io's surface. The plasma frequency $f_{p}$ (below which radio signals cannot penetrate) is given by $f_{p}=8.98 \mathrm{~Hz} \times \sqrt{N_{e}}$, where $N_{e}$ is the electron density in units of $\mathrm{m}^{-3}$. These profiles have been measured using radio occultation measurements ${ }^{20,21}$, resulting in an Io plasma frequency of $2.2 \mathrm{MHz}$. The peak electron number density at Io goes down at night to $9 \times 10^{9} \mathrm{~m}^{-3}$, which would take the plasma frequency down to $0.85 \mathrm{MHz}$ at night. In practice, the observations will need to be $1-2 \mathrm{MHz}$ above that to account for absorptive, dispersive, and birefringent effects near the cutoff frequency. Using the dayside 2 $\mathrm{MHz}$ cutoff frequency and adding $2 \mathrm{MHz}$ to account for additional effects and $1 \mathrm{MHz}$ of margin, a reasonable frequency for passive sounding is $5 \mathrm{MHz}$. A passive receiver can cover frequencies of hundreds of $\mathrm{kHz}$ up to $35 \mathrm{MHz}$ without significantly driving the mass and volume requirements, which enables additional observation capabilities to study the total electron content of Io's ionosphere.

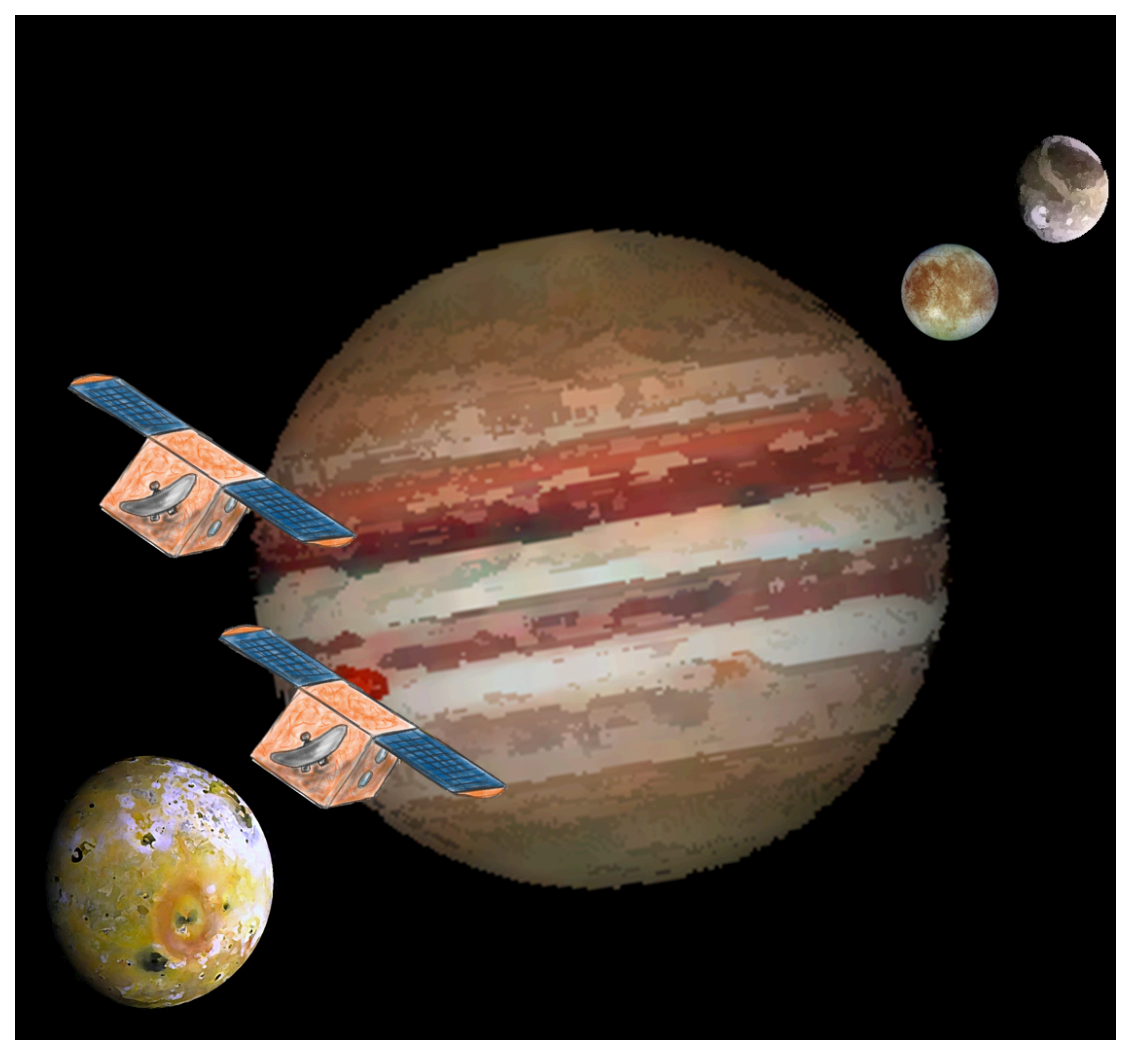

Figure 2: A design concept relying on passive radar can allow for operating multiple spacecraft simultaneously. 


\subsection{Burst frequency}

Due to the harsh radiation environment, the exploration of Io will hinge on flyby missions rather than orbiters. A key question is the probability that a Jovian burst will illuminate Io near the time of closest approach. Assuming a highly elliptical orbit with high inclination designed to avoid the Io torus, flyby speeds can be on the order of $\sim 20 \mathrm{~km} / \mathrm{s}$. At this speed, the spacecraft will displace itself a distance equivalent to the diameter of Io in 3 minutes and 20 seconds. For passive sounding to be successful, the Jovian burst duty cycle will have to be high.

To address this issue, we use data acquired by the Radio Plasma Wave Science instrument during the Cassini flyby of Jupiter. Cassini recorded the Jovian burst activity. The smallest fluxes reported ${ }^{22}$ in are $10^{-21.4} \mathrm{~W} \mathrm{~m}^{-2} \mathrm{~Hz}^{-1}$. In the vicinity of Io, this level of emission is $39 \mathrm{~dB}$ above the galactic noise floor. We will take a conservative approach and assume this value indicates that the Jovian decametric noise (DAM) source is off.

We then take the cumulative distribution function of the flux values to estimate the fraction of the time where the bursts are on. The cumulative distribution function indicates that the fraction of time the bursts are active exceeds $40-80 \%$. Note that the signal is dominated by the non-Io DAM, which is not tied to Io. These illumination opportunities should therefore apply to Io as they would

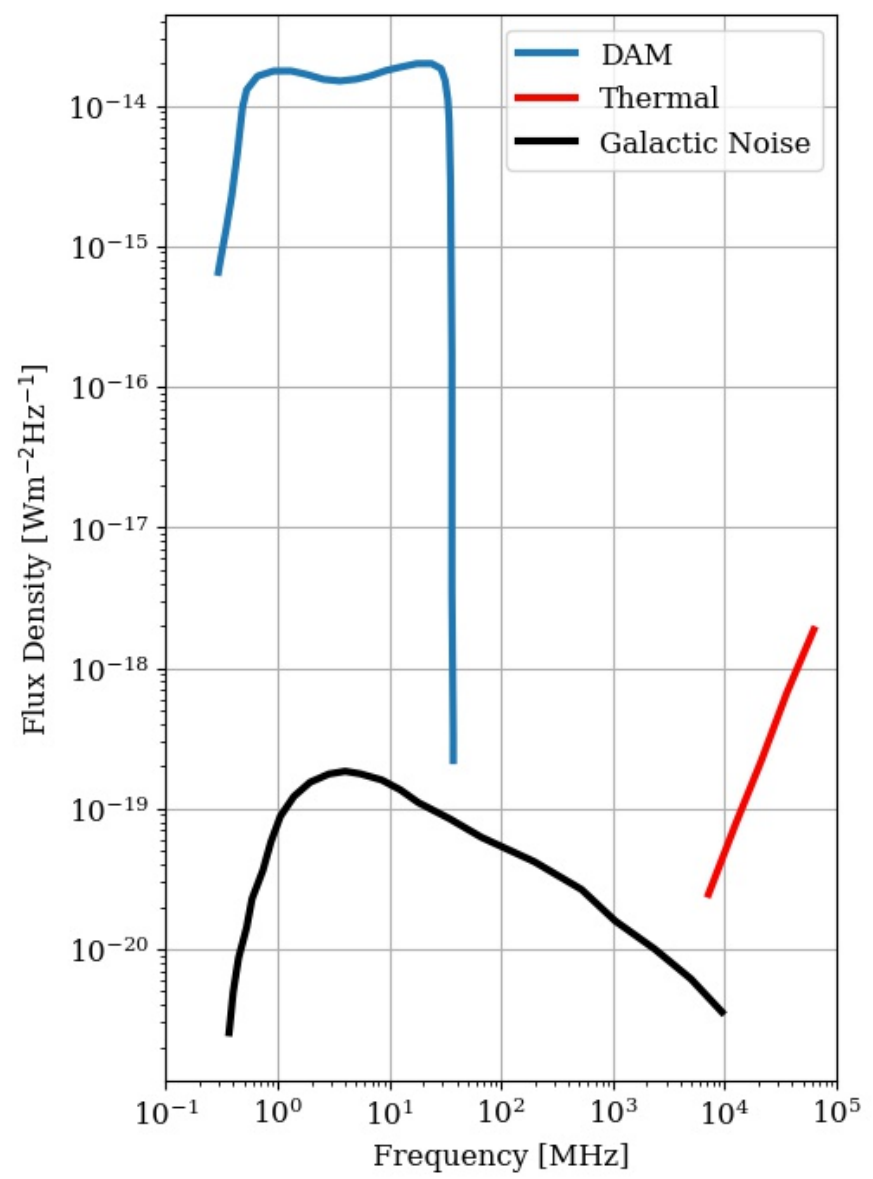

Figure 3: Radio noise sources at Io. Flux data from Cecconi et al. (2012). to any other observer.

The key to understanding the sensitivity is an estimate of the attenuation of radio signals propagating through the crust and reflected off the subsurface magma. Volcanic activity is continuously regenerating the surface of Io, so it is expected to be smooth at $100 \mathrm{~m}(3 \mathrm{MHz})$ wavelengths. For a $300 \mathrm{~km}$ altitude and $\sim 100 \mathrm{~m}$ wavelength, the Fresnel zone is 7.7 $\mathrm{km}$. For a $20 \mathrm{~km} / \mathrm{s}$ ground speed, the maximum integration time is limited to $\mathrm{t}=$ $387 \mathrm{~ms}$. The expected bandwidth is $\mathrm{BW}=$ $3 \mathrm{MHz}$ but could be as wide as $10 \mathrm{MHz}$. The time-bandwidth product results in a coherent processing gain of $60.6 \mathrm{~dB}$.

For a desired SNR of $10 \mathrm{~dB}$ for detection, the specific cross section can $>-45 \mathrm{~dB}$. Note that, in practice, the flyby produces a radargram of the subsurface interface of the $\sim 40 \mathrm{~km}$ of lateral extent expected of the chamber. For a Fresnel zone of $7.7 \mathrm{~km}$, the radargram would result in 5 independent measurements with more traces showing no reflected wave where the chamber ends. This scenario could increase the sensitivity 
to specific cross-sections $>-50 \mathrm{~dB}$. We will translate this to penetration depths in the next subsection.

\section{$\underline{\text { 5. Maximum Sounding Depth }}$}

To estimate the penetration depth of the cases discussed above, we model the subsurface reflection. Using the model by Simon et al. (2014), we estimate the maximum depth over a range of expected permittivity values and center frequencies for a conservative loss tangent $(\tan \delta)$ of 0.01 (Figure 4). Furthermore, we assume the following scenario when calculating the maximum penetration depth: (1) that we are receiving with the max integration time, (2) at a closest approach distance of $300 \mathrm{~km}$ with a flyby velocity of $20 \mathrm{~km} /$ second, (3) a Jupiter transmission event is occurring, (4) the bandwidth of the receiver is $\mathrm{BW} \leq 2(\mathrm{c} / \lambda)$, and (5) that we are receiving a reflection at nadir.
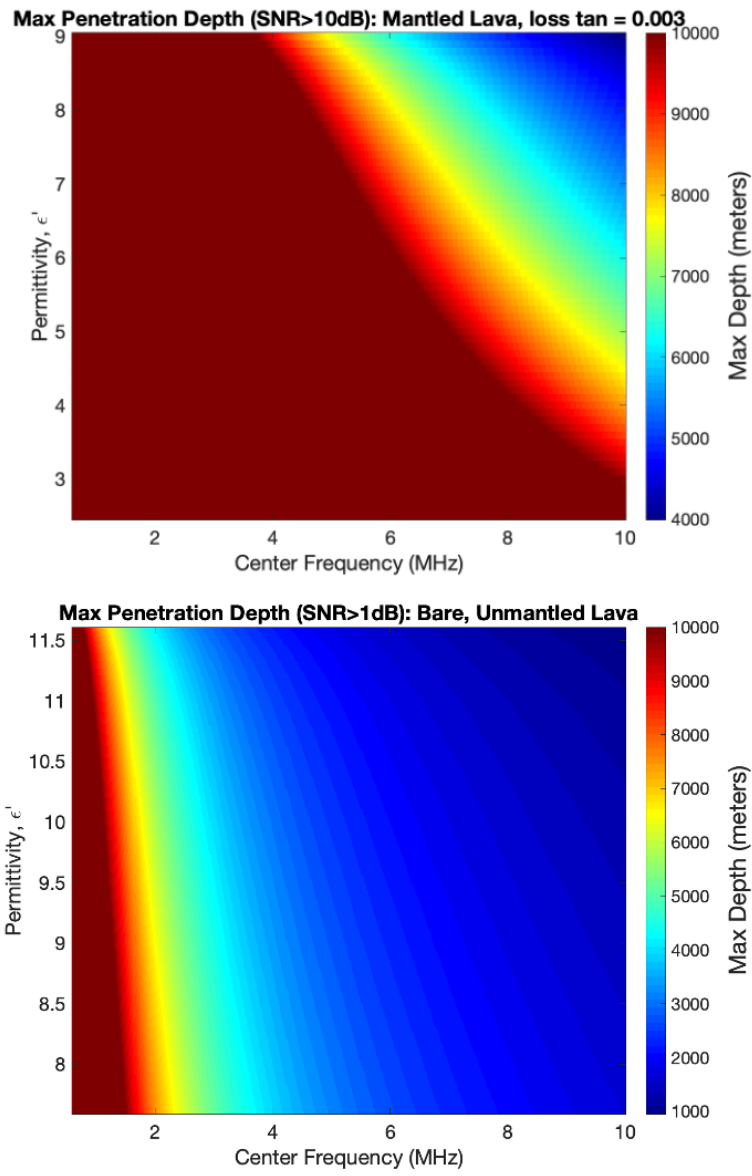
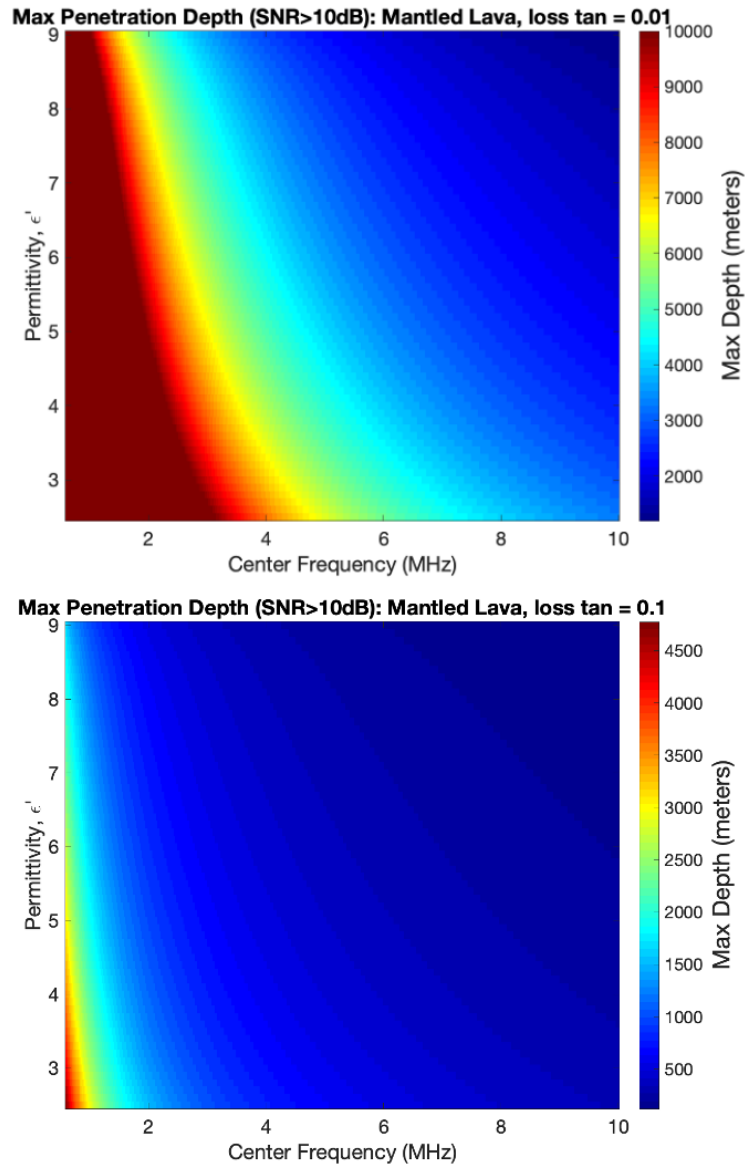

Figure 4: Maximum penetration depth as a function of center frequency and permittivity for mantled and unmantled lava using different sets of loss tangents.

Additionally, this model assumes that there are no faraday rotation losses and no ionospheric dispersion. The penetration depth depends significantly on the loss tangent and the contrast between the layered mantle and subsurface lava permittivity values. Extremely high loss tangents 
( $\tan \delta \approx 0.1$ ) would limit the penetration depths to an order of 1-3 km (not shown here), but would still allow one to examine Io's near surface; moreover, low loss tangents ( $\tan \delta \approx 10^{-3}$ ) would enable passive sounding to be used for a wide range of possible mantle permittivity values and down to $10 \mathrm{~km}$ depth. This would enable passive sounding to also search for deeper magma chambers.

\section{$\underline{\text { 6. Synthesis }}$}

We advocate the use of passive radar sounders to study Jupiter's moon Io from orbit. Passive radar is a novel approach in planetary exploration and while considered for the active sounders on the Jupiter Icy Moons Explorer (JUICE) and Europa Clipper mission, no dedicated passive sounder has been designed to date but could address outstanding scientific questions with a low-resource approach. The absence of an active transmitter when using the passive technique is particularly suitable for low mass, low power mission concepts. The strong radio noise environment allows for the use of robust, low-power receivers and a simple antenna design, making the concept ideal for challenging targets. The main remaining challenges are to explore how the complex geometry and frequency of Jupiter's radio noise emissions can be best used to maximize the scientific return while minimizing the amount of required spacecraft.

Advancing this technology will be of significant use in the further development of passive radar sounders, which have the potential to be flown as self-standing, low-resource missions or complement more complex missions as a low-power asset. The results will therefore not be limited to solely this application within the Jovian system but can be used in combination with other radio noise sources, such as the Sun, to enable further compelling mission concepts within the inner Solar System.

\section{References:}

${ }^{1}$ Keane et al. (2020), white paper The case for Io exploration, ${ }^{2}$ Keane et al. (2020), Recommendations for Addressing Priority Io Science in the Next Decade: Missions, Technology, Instruments, Earth-based Observations, Research and Analysis, Workforce, and More, ${ }^{3}$ de Kleer, et al. Tidal Heating: Lessons from Io and the Jovian System, Final Report for the Keck Institute for Space Studies (2019). ${ }^{4}$ Peale et al. 1979, https://doi.org/10.1126/science.203.4383.892. ${ }^{5}$ Tackley et al. 2001, https://doi.org/10.1006/icar.2000.6536. ${ }^{6}$ Veeder et al. (2012), https://doi.org/10.1016/j.icarus.2012.04.004. ${ }^{7}$ Hamilton et al. (2013), https://doi.org/10.1016/j.epsl.2012.10.032. ${ }^{8}$ Davies et al. Journal of Volcanology and Geothermal Research (2010), https://doi.org/10.1016/j.jvolgeores.2010.04.009. ${ }^{9}$ Davies et al., Icarus, (2006), Pages 460-477, https://doi.org/10.1016/j.icarus.2006.05.012. ${ }^{10}$ Radebaugh et al. (2001), JGR., 106, https://doi.org/10.1029/2000JE001406. ${ }^{11} \mathrm{McEwen}$ et al. (2000), Science, https://doi.org/10.1126/science.288.5469.1193 ${ }^{12}$ Schenk and Bulmer (1998) Science, https://doi.org/10.1126/science.279.5356.1514. ${ }^{13}$ Bland and McKinnon (2016), https://doiorg.stanford.idm.oclc.org/10.1038/ngeo2711. ${ }^{14}$ Battaglia et al., Icarus (2014) https://doi.org/10.1016/j.icarus.2014.03.019 ${ }^{15}$ Hartogh and Ilyushin, (2016), https://doi.org/10.1016/j.pss.2016.05.008. ${ }^{16}$ Schroeder et al. (2016), https://doi.org/10.1016/j.pss.2016.10.007.

${ }^{17}$ Romero-Wolf et al. (2015), https://doi.org/10.1016/j.icarus.2014.10.043. ${ }^{16}$ Romero-Wolf et al., (2016), https://doi.org/10.1016/j.pss.2016.06.010. ${ }^{18}$ Peters et al. (2018), doi.org/10.1109/TGRS.2018.2850662 ${ }^{19}$ Ellingson (2005), https://doi.org/ 10.1109/TAP.2005.852281. ${ }^{20}$ Kasper et al. (2019), https://doi.org/10.1109/AERO.2019.8742146 ${ }^{21}$ Kliore et al. (1975), https://doi.org/10.1016/0019-1035(75)90057-3. ${ }^{22}$ Hinson et al. (1998), https://doi.org/10.1029/98JA02659. 